\title{
Rectal Bleeding in a Young Patient with Cavernous Hemangioma and Bone Hypertrophy
}

\author{
Abdol Rahim Masjedizadeh ${ }^{1,2^{*}}$, Pezhman Alavinejad ${ }^{1,2}$, Seyed Jalal Hashemi ${ }^{1,2}$
}

1. Research Institute for Infectious Diseases of the Digestive System, Ahvaz Jundishapur University of Medical Sciences, Iran

2. Department of Internal Medicine, Ahvaz Jundishapur University of Medical Sciences, Iran

\footnotetext{
* Corresponding Author:

Abdolrahim Masjedizadeh, MD

Research Institute for Infectious Diseases of the Digestive System, Division of Gastroenterology and Herpetology, Department of Internal Medicine, Ahvaz Jundishapur University of Medical Sciences, Iran Telfax: + 986133910642

Email: Rahim.masjedi@gmail.com Received: 18 Aug. 2015

Accepted: 10 Oct. 2015
}

\section{Please cite this paper as:}

Masjedizadeh AR, Alavinejad P, Hashemi SJ. Rectal Bleeding in a Young Patient with Cavernous Hemangioma and Bone Hypertrophy. Middle East J Dig Dis 2015;8:73-4. DOI :10.15171/mejdd.2016.11

A 22-year-old man with history of intermittent rectal bleeding since infancy referred for colonoscopy. His vital signs and lab results included blood pressure: 120/80, pulse rate: 102 , hemoglobin $9 \mathrm{~g} / \mathrm{dL}$, hematocrit $27 \%$, and serum ferritin $7 \mu \mathrm{g} / \mathrm{L}$. On physical examination, he was pale with a slightly raised deep violet colored lesion ("port-wine") on the flank and cavernous hemangiomas with a verrucous quality around the umbilicus and trunk (figures A, and B). Soft tissue and bone hypertrophy with fused or extra toes were also present on the feet (figure C).

Colonoscopy revealed diffuse cavernous hemangioma with tortuous dilated blood vessels, elevated red-wine nodules with poorly defined margins and normal mucosa between affected areas extending from lower rectum to sigmoid (figures D, E). The radial ultrasonography of rectum showed submucosal vascular signal (figure F).
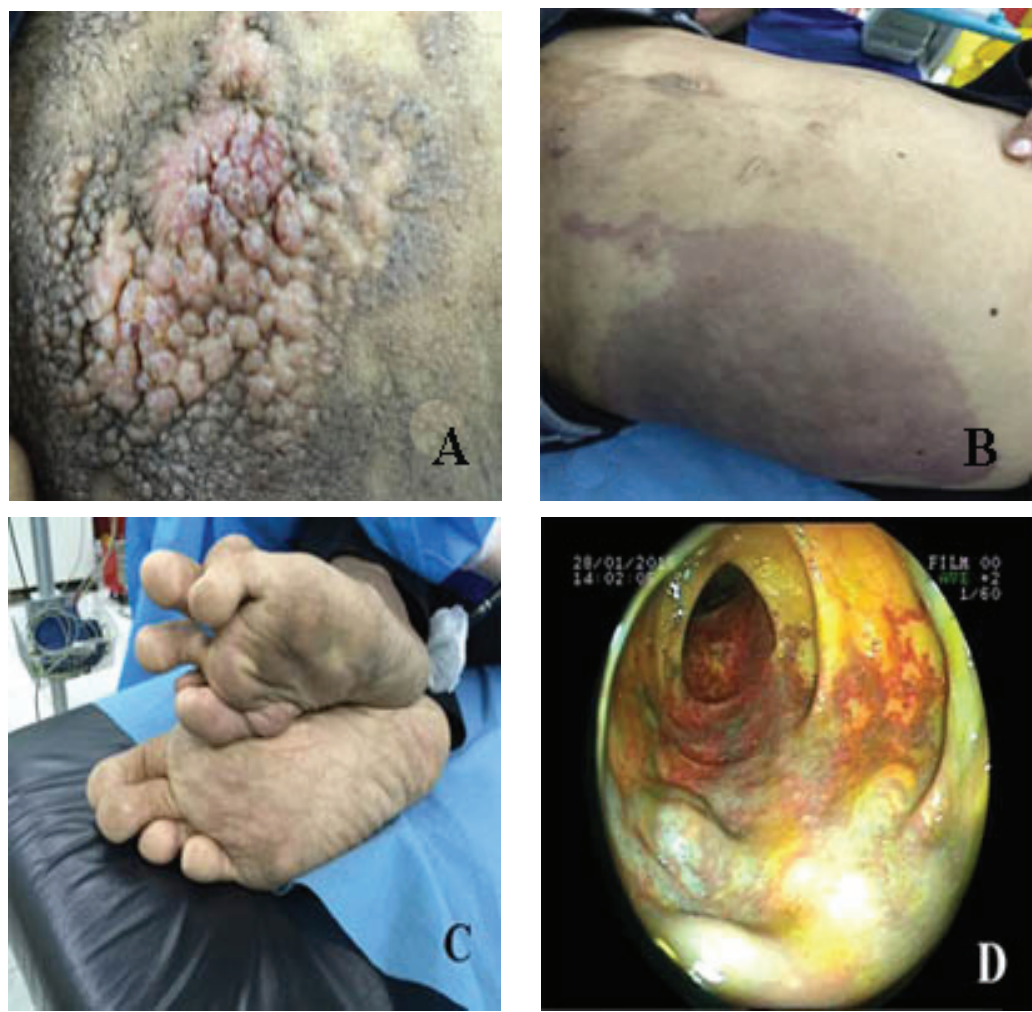


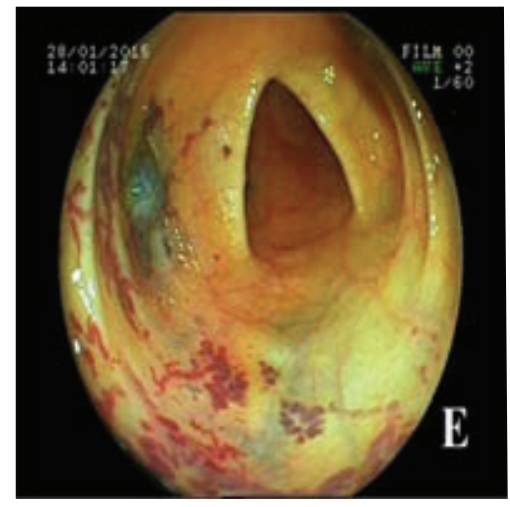

What is your diagnosis?

Answer:

The clinical triad of capillary malformation, soft tissue and bone hypertrophy of an extremity, and varicose veins or venous and lymphatic malformations is compatible with Klippel-Trénaunay syndrome (KTS). The diagnosis of KTS is usually made when any two of the three features are present. KTS prevalence is about 1: 100,000. ${ }^{1}$ It affects female and male patients equally and presents at birth or during early infancy or childhood.

The exact cause of KTS is unknown; although several theories exist including intrauterine damage to the sympathetic ganglia. Some researchers believe that deep vein abnormalities lead to venular hypertension, development of varices, and limb hypertrophy. Most cases are sporadic, although an autosomal dominant pattern of inheritance has been reported. ${ }^{2}$

KTS generally affects a single extremity and the leg is the most common site of involvement followed by the arms, trunk, and rarely head and neck. Most patients demonstrate all the three signs of the clinical syndrome.

Rectal and bladder hemorrhage are serious complications of pelvic vascular malformation and have been reported in $1 \%$ of cases. The gastrointestinal tract is involved in $20 \%$ of patients. ${ }^{3}$ Bleeding is the most common symptom, which ranges from occult bleeding to massive, life-threatening hemorrhages. The most frequently reported sites of bleeding are the distal colon, and rectum. Jejunal hemangiomas are usually the origin of upper gastrointestinal bleeding. ${ }^{4}$

Treatment for KTS is conservative. Symptomatic cavernous hemangiomas of colon are usually treated surgically by resection of the involved segment and

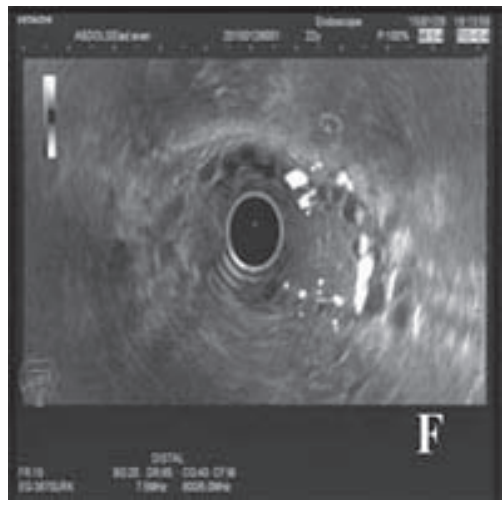

permanent colostomy. When diffuse colonic hemangiomas are associated with KTS, surgery is complicated because of extensive visceral involvement; hence radiotherapy could be a more suitable option.

Therapeutic endoscopy with Argon plasma coagulation has been offered for localized cavernous hemangioma but with unpredictable results. ${ }^{5}$ Our patient refused our advice for surgery and accepted a conservative supportive care and follow-up.

\section{CONFLICT OF INTEREST}

The authors declare no conflict of interest related to this work.

\section{REFERENCES}

1. Lee MS, Liang MG, Mulliken JB. Diffuse capillary malformation with overgrowth: a clinical subtype of vascular anomalies with hypertrophy. $J$ Am Acad Dermatol 2013;69:589-94. DOI:10.1016/j.jaad.2013.05.030. Epub 2013 Jul 29.

2. Oduber CE, Young-Afat DA, van der Wal AC, van Steensel MA, Hennekam RC, van der Horst CM. The persistent embryonic vein in Klippel- Trenaunay syndrome. Vasc Med 2013;18:185-91. DOI:10.1177/1358863X13498463.

3. Deepinder F. GI bleeding, colon varicosities, and visceral enlargement as a manifestation of Klippel-Trenaunay syndrome. Clin Gastroenterol Hepatol 2011;9:e126-7.

4. Thosani N, Ghouri Y, Shah S, Reddy S, Arora G, Scott LD. Life-threatening gastrointestinal bleeding in Klippel-Trenaunay syndrome. Endoscopy. 2013;45 Suppl 2 UCTN:E206. DOI:10.1055/s-0032-1326645.

5. Herman R,Kunisaki S, Molitor M, Gadepalli S, R. Dillman J , Geiger J. Rectal bleeding, deep venous thrombosis, and coagulopathy in a patient with Klippel-Trénaunay syndrome. J Pediatr Surg 2012;47:598-600. DOI:http:// dx.doi.org/10.1016/j.jpedsurg.2011.12.016.

6. and coagulopathy in a patient with Klippel-Trénaunay syndrome. J Pediatr Surg 2012;47:598-600. 